$1.26 ; 1.07,1.49)$. The added predictive value was higher in the group that experienced financial strain, in particular for CRP.

Conclusion The positive association between inflammatory biomarkers and CVD death was much stronger in the presence of financial strain. When assessing the presence of inflammation with a single test for prediction of CVD risk, it may be necessary to take into account the presence of chronic psychosocial stress.

\section{OP44 \#IDEAL CARDIOVASCULAR RISK PROFILES AND AGEING: EVIDENCE FROM 421,000 OLDER PERSONS IN TWO COHORTS}

${ }^{1} J \mathrm{~L}$ Atkins*, '1 Delgado, ' $\mathrm{LC}$ Pilling, 'K Bowman, ${ }^{1} \mathrm{JAH}$ Masoli, ${ }^{2} \mathrm{GA}$ Kuchel, ${ }^{3} \mathrm{~L}$ Ferrucci,

${ }^{1,2}$ D Melzer. 'Epidemiology and Public Health Group, University of Exeter Medical School, Exeter, UK; ${ }^{2}$ Center on Aging, University of Connecticut, Connecticut, USA; ${ }^{3}$ National Institute on Aging, Baltimore, USA

\subsection{6/jech-2018-SSMabstracts.44}

Background Individuals with low cardiovascular risk factor profiles experience lower rates of cardiovascular diseases (CVD) and mortality. However, little is known about how older persons with near ideal cardiovascular risk factors age, especially for non-cardiovascular outcomes. We tested whether older individuals with near ideal CVD risks were less likely to develop ageing-related adverse health outcomes in two large cohorts.

Methods Data were from population representative primary care medical records (Clinical Practice Research Datalink, CPRD, England, $n=239,591$ ) and also healthy volunteers (UK Biobank, UKB, $n=181,820$ ), aged 60 to 69 years at baseline followed for up to 10 years. A cardiovascular risk score (CRS) summarized smoking status, low density lipoprotein cholesterol (LDL), blood pressure, body mass index (BMI), fasting glucose and physical activity, grouping individuals as low (all factors near ideal), moderate or high CRS, following the American Heart Association 'Life's Simple 7' approach. Data were available on a range of prevalent and incident ageing-related adverse health outcomes from CPRD and UK Biobank. Logistic regression and Cox proportional hazards regression models (Fine and Grey models including death as a competing risk) were used to test associations between the CRS and prevalent or incidence health outcomes respectively.

Results Older subjects with near ideal CVD risks had less chronic pain (UKB baseline odds ratios (OR), 0.52, 95\% CI 0.50 to 0.54 ), lower incidence of incontinence (CPRD subhazard ratio (sub-HR), $0.75,95 \%$ CI 0.63 to 0.91), falls (subHR, 0.82 , 95\% CI 0.73 to 0.91 ) and fragility fractures (subHR, 0.78 , 95\% CI 0.65 to 0.93 ). Only $5.4 \%$ in our primary care sample with near ideal CVD risks became frail (Rockwood index) versus $24.2 \%$ with high risks. All-cause mortality was markedly lower in the low CRS group (vs. high CRS, HR, 0.40: 95\% CI 0.35 to 0.47). All associations showed dose-response relationships. Estimates of associations were remarkably similar in both cohorts despite differences in the cohort profiles and risk factor ascertainment, suggesting robustness of results.

Conclusion Persons aged 60 to 69 years with near ideal cardiovascular risk factor profiles have substantially lower incidence of common conditions of ageing, including frailty. Optimizing
CVD risk factors may substantially reduce the burden of morbidity in later life: we thus likely already have the tools for radical improvement in ageing health outcomes. However, further work is needed to provide evidence of whether this association is causal. Using largescale data from UK Biobank, we aim to expand the existing study and tease apart the roles of component risk factors using genetic evidence.

\section{OP45 ALLOSTATIC LOAD AND EFFORT-REWARD IMBALANCE: ASSOCIATIONS OVER THE WORKING-CAREER}

${ }^{1} \mathrm{Il}$ Cuitun Coronado*, ${ }^{1} \mathrm{~T}$ Chandola, ${ }^{2} \mathrm{~A}$ Steptoe. ${ }^{1}$ Cathie Marsh Institute and Social Statistics, University of Manchester, Manchester, UK; ${ }^{2}$ Research Department of Behavioural Science and Health, University College London, London, UK

\subsection{6/jech-2018-SSMabstracts.45}

Background Although associations between work stressors and stress-related biomarkers have been reported in cross-sectional studies, the use of single time measurements of work stressors could be one of the reasons for previously inconsistently reported associations. This study examines whether repeated reports of work stress towards the end of the working career predicts allostatic load, a measure of chronic stress related physiological processes.

Methods Data from waves 2 to 6 of the English Longitudinal Study of Ageing (ELSA) were analysed, with a main analytical sample of 2663 older adults (aged 50+) who had at least one measurement of effort-reward imbalance between waves 2-6 and a measurement of allostatic load at wave 6. From this main analytical sample, a subsample of 1020 respondents had their allostatic load measured at wave 2. Cumulative work stress over waves 2-6 were measured by the effort-reward imbalance model. Negative binomial regression models were used to estimate the association between effort-reward imbalance and allostatic load after controlling for covariates (categorized age, gender, ethnicity, smoking status, general health, number of medications used, depressive symptoms using the Centre for Epidemiologic Studies Depression Scale, physical activity, and alcohol use in the last 12 months).

Results Employees with effort-reward imbalance at the more recent waves $5(0.09,-0.002-0.17)$ and $6(0.13,0.03-0.22)$ had higher levels of wave 6 allostatic load compared to those who did not report any imbalance at those waves. The predicted levels of allostatic load by cumulative reports of effortreward imbalance from the model controlling for wave 2 allostatic load showed that workers who reported two or more occasions of effort-reward imbalance had a higher estimate of the allostatic load index $(0.11$, 95\% CI 0.01 to 0.22$)$ compared to workers who never reported effort-reward imbalance. Conclusion The study finds some evidence that older adults aged $50+$ living in England who repeatedly reported work related stressors had higher levels of the allostatic load index than those who did not report any effort-reward imbalance. This association was robust to controlling for a range of potential health and socio-demographic confounders, as well as baseline levels of allostatic load. The findings of a doseresponse association between effort-reward imbalance and allostatic load, as well as the timing of the stressor and stress response, suggest that exposure to work-related stressors may have adverse consequences for physiological health through increasing adverse levels of stress related biomarkers. 